\section{Quality Oral Health Care for the Elderly Population: An Ac- ademic and Patient-Awareness Perspective}

\author{
Pollene Speed Mclntyre* \\ Department of Restorative Dentistry, University of Washington, USA
}

As dentists, our first response to the public should be to serve as effectual and compassionate health care providers. This role is one that includes each of us taking the necessary steps to ensure that all populations are provided feasible and affordable quality oral health care. This challenge requires even more individual commitment when we consider the tasks necessary to develop policies and procedures, create training guidelines and establish appropriate facilities to provide this care. It is a challenge that is intensified particularly in caring for and treating some of our most venerably population, that being the elderly socially, economically and physically dependent and independent patients.

According to the 2010 census, the US population 65 and older is now the largest in terms of size and percent of the population. The group grew at a faster rate than the total population between 2000 and 2010. The 2010 census determined that there were 40.3 million people 65 and older on April 1, 2010, increasing by 5.3 million since the 2000 census when this population numbered 35.0 million. The population of those 65 and older grew at 15.1 percent while the total population grew at 9.7 percent. More so according to the US administration on aging, the population of Americans older than 65 years is expected to double to about 71 million by 2040 . Along the same lines the population of Americans older than 85 years is expected to increase to 9.6 million by 2030 . Numerous academic papers have noted that the dental needs of these patients have increased given that more individuals are keeping their teeth much longer with much more involved dental restorative needs. Thru conversation with peers, it is clear that many general dentists are seeing an increase in dental cosmetic, social and functional expectations of this population. In that it is not unusual for a 70-80 year old patient to request comprehensive restorative treatment plans options with the

${ }^{*}$ Corresponding author: Pollene Speed Mclntyre, Department of Restorative Dentistry, University of Washington, USA, Tel: +1 2065435948; E-mail: speedp@uw.edu

Citation: McIntyre SP (2015) Quality Oral Health Care for the Elderly Population: An Academic and Patient-Awareness Perspective. J Geronto Geriatr Med 1: 002

Received: August 01, 2015; Accepted: August 03, 2015; Published: August 18, 2015 expectation that they will need their teeth for many more years. While conversely, the population of dental professionals willingly skillful to provide this level of specialized dentistry is somewhat limited and at the very least the number of available dental providers to perform this work is unclear.

As a matter of fact, a recent review of dental specialists as identify by the US Dental School programs does not include Geriatric dentistry as a specialty. Paralleling this is the resultant workforce of available dentists who are properly trained for this consistently changing clinical and technical work. In order to provide quality oral care to the elderly population we must first identify them as a priority group that is in need of specialized oral health care. This declaration will lead to the establishment of guideposts for educational and practical outcomes generally and specifically for the establishment of dental training facilities designed to treat these patients.

Withstanding this formal identification of a population in need, a systematic academic and patient - awareness process of addressing this challenge should include dental school admissions programs establishing criteria that will help create a pool of applicants with a demonstrated commitment and thus more likely to work with the elderly population. The establishment of a Geriatric dentistry core curriculum that focus on didactic and chair side training of students must become a priority. As well as the proper training of current and future dental students, the dental profession must create selective and quality resources of continuing dental education training for the general dentistry professional. While the utilization of currently practicing general dentists to provide these needed dental services seems like a reasonable solution, the proper avenue to address this challenge is to develop appropriate and formal standards within our educational institutions. These programs should be specifically designed to train current students as well as be a reliable resource of training for all practicing general dentists to become clinically competent to serve these patients. These clinical standards should include not only upgraded clinical technique and procedures for establishing and maintaining a quality, functioning and healthy oral environment, restoring existing dental restorations or aggressive root caries treatment and management but also exploring progressive treatment plans that will properly serve these patients. These upgraded standards developed with the oversite of our National Dental Accreditation body should be embraced by organized dental organizations such as our national and local dental societies.

As a dental profession, we cannot wait to be instructed and guided by other entities to make these changes, this is a challenge that our profession must stop and do the necessary preparatory work so that we may better serve all of our patients as the compassionate and competent health care providers that we are entrusted and should thus be answerable. 